\title{
Towards Intelligent Miniature Flying Robots
}

\author{
Samir Bouabdallah ${ }^{1}$ and Roland Siegwart ${ }^{2}$ \\ 1 Autonomous Systems Lab, EPFL samir.bouabdallah@epfl.ch \\ 2 Autonomous Systems Lab, EPFL roland.siegwart@epfl.ch
}

\begin{abstract}
Summary. This paper presents a practical method for small-scale VTOL ${ }^{3}$ design. It helps for elements selection and dimensioning. We apply the latter to design a fully autonomous quadrotor with numerous innovations in design methodology, steering and propulsion achieving 100\% thrust margin for $30 \mathrm{~min}$ autonomy. The robot is capable of rotational and translational motion estimation. Finally, we derive a nonlinear dynamics simulation model, perform a simulation with a PD test controller and test successfully the robot in a real flight. We are confident that "OS4" is a significant progress towards intelligent miniature quadrotors.
\end{abstract}

Key words: VTOL design, quadrotor, quadrotor modelling

\section{Introduction}

Research activities in rolling robots represent the lion's part in mobile robotics field. In the case of complex or cluttered environments the miniature flying robots emphasis all their advantages. The potential capabilities of these systems and the challenges behind are attracting the scientific community [1], [2], [3], [4]. Surveillance, search and rescue in hazardous cluttered environments are the most important applications. Thus, vertical, stationary and slow flight capabilities seem to be unavoidable making the rotorcraft dynamic behavior a significant pro. In cluttered environments the electrical propulsion, the compactness, the hard safety and control requirements, the abandon of GPS are not only a choices, they are imposed. Most of the early developments suffer from a lack of intelligence, sensory capability and short autonomy except for the larger machines. In this paper we present the new design of a fully autonomous quadrotor helicopter named "OS4", equipped with a set of sensors, controllers, actuators and energy storage devices enabling various scientific experiments. This robot was built following a design methodology adapted for miniature VTOL systems.

\footnotetext{
${ }^{3}$ Vertical Take-Off and Landing
} 


\section{Design}

The interdependency of all the components during the design phase makes the choice of each one strongly conditioned by the choice of all the others and vice-versa.

\subsection{Design Methodology}

The open-loop simulation analysis [5] have shown clearly the strong dynamic instability of a quadrotor. However, one can improve the stability by simply acting on several system parameters. For instance, spreading the mass in each of the four propulsion groups ${ }^{4}(\mathrm{PG})$ increases the diagonal elements of the inertia matrix. Moreover, building the quadrotor in a regular cross configuration simplifies the control law formulation [6]. One can also optimize the vertical distance between the $\mathrm{CoG}$ and the propellers center in order to increase the damping (CoG below propellers), or slow the natural frequency [7] (CoG above propellers). On the other hand, augmenting the horizontal distance (CoG-propellers) increases the inertia. Taking a decision concerning all these design variables requires to follow an appropriate methodology. This paper proposes a practical method to handle the design problematic of a small scale rotorcraft by combining the theoretical knowledge of the system and a minimum of optimization results analysis. This method is by far less complex than a traditional $\mathrm{MDO}^{5}$.

\section{The General Method}

The starting point of the design process is to define an approximate target size and weight of the system, dictated generally by the final application. This gives a good idea about the propeller size to use. Using an analytical model of a propeller with for instance blade element theory or by an experimental characterization of a given propeller [8] one can estimate the thrust and drag coefficients which permits the verification of the thrust requirements. For the special case of the quadrotor a rule of thumb fixes an optimum thrust to weight ratio to $2: 1^{6}$. This was observed during several simulations and experienced with the limited actuators of the first "OS4" prototype [8]. The propeller's information helps to build a selected actuators data bank which are likely to meet the power requirements. Then, a rough estimation of the airframe and avionics masses is necessary (see Fig. 6) to have a first estimation of the total mass without battery. The latter is found by an iterative algorithm as schematized in Fig. 1.

\footnotetext{
${ }^{4}$ Propeller + Gearbox + Motor

${ }^{5}$ Multidisciplinary Design Optimization

${ }^{6}$ 1.4:1 for a miniature coaxial and 4:1 for small scale aerobatic helicopters
} 


\section{The Iterative Algorithm}

The process starts by picking-up an actuator from the data bank, estimating it's performances with the propeller's model, computing the system total mass, power consumption, propulsion group cost and quality factors in the equilibrium and maximum thrust points. Moreover, the autonomy and a special index (autonomy/mean power) characterize the overall system quality. This is done for an incremental battery mass variable, for every actuator in the data bank as schematized in Fig. 1.

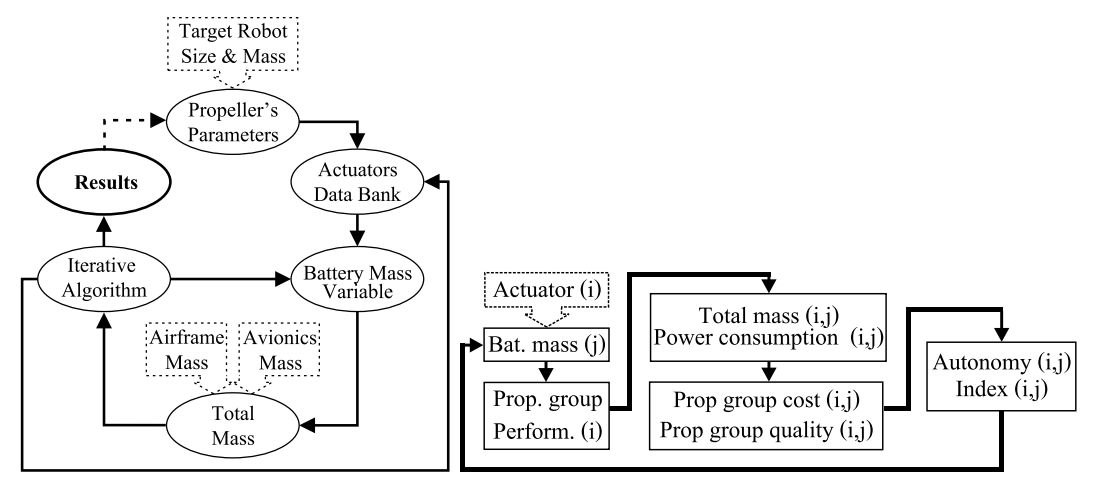

Fig. 1. Left:The design method flowchart. Right:The iterative algorithm flowchart.

\section{2 "OS4" Quadrotor Design}

The "OS4" quadrotor developed during this project represents a design example following the method described in Subsec. 2.1. The targeted system is about $500 \mathrm{~g}$ in mass and $800 \mathrm{~mm}$ in span.

\section{The Propulsion Group Design}

The "OS4" requirements lead to a $300 \mathrm{~mm}$ diameter propeller. The main design variables of a PG are listed in Table 1, and used in the models in Table 2. Finally, the choice of the PG components was based on the iterative algorithm classification with an average cost factor of $\mathbf{C}=0.13 \mathrm{~W} / \mathrm{g}$ and a quality factor of about $\mathbf{Q}=5 \mathrm{~g} / \mathrm{W}$. This was for a given Lithium-polymer battery mass of $m_{\text {bat }}=230 \mathrm{~g},(11 \mathrm{~V}, 3.3 \mathrm{Ah})$ and an autonomy estimation of 30 minutes. The choice of 2 blades propeller topology rather than more is mainly due to loss of motor efficiency and large rotor inertias with a heavier propeller. The latter is made out of carbon and was adapted to our specifications. The electrical motors torque in these application being limited, the gearbox seem to be mandatory and beneficial for such VTOL to preserve good motor efficiency. 
Table 1. Propulsion group design variables

\begin{tabular}{|c|c|c|c|c|c|c|c|c|c|c|c|}
\hline propeller & & OS4 & unit & gearbox & & OS4 & unit & motor & & OS4 & unit \\
\hline efficiency & $\eta_{p}$ & $62-81$ & $\%$ & efficiency & $\eta_{g b}$ & 96 & $\%$ & efficiency & $\eta_{m}$ & |50-60| & $\%$ \\
\hline mass & $m_{p}$ & 5.2 & $\mathrm{~g}$ & mass & $m_{g b}$ & 7 & $\mathrm{~g}$ & mass & $m_{m}$ & 12 & $\mathrm{~g}$ \\
\hline thrust coef. & $b$ & $3.13 \mathrm{e}-5$ & $\mathrm{Ns}^{2}$ & $\max$ torque & & 0.15 & $\mathrm{Nm}$ & max. power & $P_{e l}$ & 35 & W \\
\hline drag coef. & $d$ & $7.5 \mathrm{e}-7$ & $\mathrm{Nm} \mathrm{s}^{2}$ & max. speed & & 1000 & $\mathrm{rad} / \mathrm{s}$ & internal res. & $\mathrm{R}$ & 0.6 & $\Omega$ \\
\hline inertia & $J_{r}$ & $6 e-5$ & $\mathrm{~kg} \cdot \mathrm{m}^{2}$ & inertia & $J_{g b}$ & $1.3 \mathrm{e}-6$ & $\mathrm{~kg} \cdot \mathrm{m}^{2}$ & inertia & $J_{m}$ & $4 e-7$ & $\mathrm{~kg} \mathrm{~m}^{2}$ \\
\hline speed & $\Omega$ & 199-279 & $\mathrm{rad} / \mathrm{s}$ & red. ratio & $\mathrm{r}$ & $4: 1$ & & torque cst. & $\mathrm{k}$ & 5.2 & $\mathrm{mN} \mathrm{m} / \mathrm{A}$ \\
\hline
\end{tabular}

Table 2. Propulsion group component's models. $\mathbf{T}_{\mathbf{w}}$ and $\mathbf{B W}$ (max. control frequency) are respectively the thrust/weight ratio and the PG bandwidth (see tab:PGDesignVariables for symbols definitions)

\begin{tabular}{|c|c|}
\hline component| & model \\
\hline Propeller & $\mathbf{b}, \mathbf{d} \times \mathbf{\Omega}^{2}=\mathbf{T}, \mathbf{D}$ \\
\hline Gearbox & $\mathbf{P}_{\text {in }} \times \eta_{\mathrm{gb}}=\mathbf{P}_{\text {out }}$ \\
\hline $\begin{array}{l}\text { DC motor } \\
\text { PG cost }\end{array}$ & $\begin{aligned}-\frac{\mathrm{k}^{2}}{\mathrm{R}} \boldsymbol{\omega}-\mathbf{D}+\frac{\mathrm{k}}{\mathrm{R}} \mathbf{u} & =\mathbf{J} \frac{\mathrm{d} \omega}{\mathrm{d} \mathbf{\omega}} \\
\mathbf{P}_{\mathrm{el}} /\left(\mathbf{T}-\mathbf{m}_{\mathbf{p g}}\right) & =\mathbf{C}\end{aligned}$ \\
\hline PG quality & $\mathbf{T}_{\mathbf{w}} \times \mathbf{B W} / \mathbf{\Omega} \times \mathbf{C}=\mathbf{Q}$ \\
\hline
\end{tabular}

This is linked to the fact that we prefer to use large and low speed propellers. The high power/weight ratio of the selected (12 g, 35W) BLDC motor justifies this choice even with the control electronics included. A $6 \mathrm{~g}$ MCU based $\mathrm{I}^{2} \mathrm{C}$ controller was specially designed for the sensorless outrunner LRK195.03 motor as shown in Fig. 2. Obviously, BLDC motors offer high life-time and less electromagnetic noise. The ready to plug PG weights $40 \mathrm{~g}$ and lifts more than $260 \mathrm{~g}$.

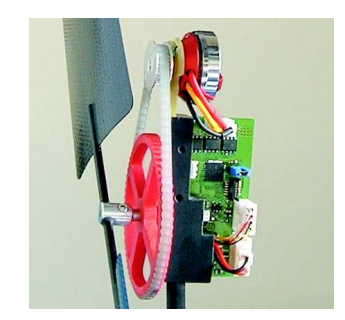

Fig. 2. The "OS4" propulsion group. 


\section{The Avionics}

The limited payload imposes some restrictions on the sensors. For yaw angle and linear displacements measuring on "OS4" we use a lightweight vision based sensor. Fig. 3 represents the block diagram of the "OS4" avionics.

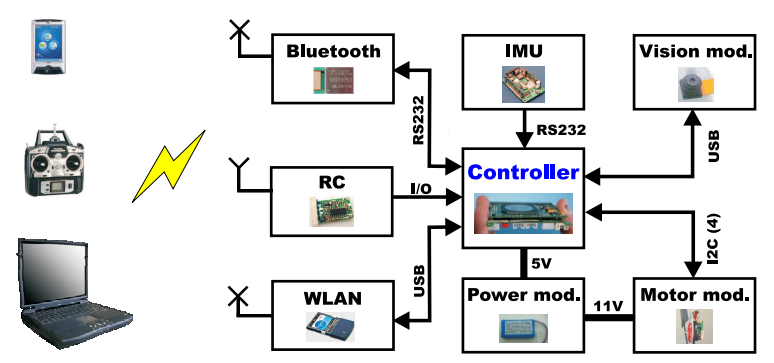

Fig. 3. OS4 block diagram.

The Inertial Measurements Unit

The "OS4" quadrotor uses the MT9-B, a $15 \mathrm{~g}$ (OEM) commercially available IMU to get absolute roll and pitch angles and their corresponding angular velocities at up to $512 \mathrm{~Hz}$. The IMU is installed horizontally at $45 \mathrm{deg}$ from the carbon rods. In this configuration the robot flies forward following the IMU $x$ axis. This original quadrotor steering makes it possible to reduce the lift dissymmetry effect as showed in Fig. 4.

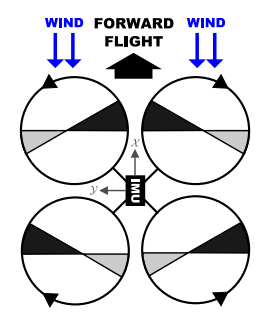

Fig. 4. Reducing the lift dissymmetry effect. Black region:High lift, Grey region:Low lift.

\section{The Vision Module}

The GPS signal weakness and precision in cluttered environments makes it difficult to use. On the other hand, the surrounding metallic structures strongly disturb the IMU magnetic based yaw estimation. Thus, it was necessary to 
develop a lightweight visual positioning module, assuming a flat floor with chessboard structure. The system uses a $0.6 \mathrm{~g}$ micro-camera (OV7648) to extract and track the chessboard corners and the roll and pitch information to correct the motion estimation. It is presently possible to provide the relative altitude, the yaw angle, the linear horizontal displacements and their corresponding time derivatives at up to $15 \mathrm{~Hz}$. The precision is of the order of the tenth of degree for the yaw, millimeter for the altitude and centimeter for the horizontal displacements. Obviously, the error grows with the displacement speed while the sensor is valid for roll and pitch angles of $\pm 20 \mathrm{deg}$. Considering chessboard squares of $40 \mathrm{~mm}$ side, the altitude measurement range is $0.5 \mathrm{~m}$ to $3 \mathrm{~m}$. It was thus necessary to add a laser diode and to extract it's spot position in the image estimating the altitude for the take-off and landing procedures. The actual module is a preliminary approach. The final goal is to achieve a visual odometry without modifying the environment.

\section{The Controller}

Embedding the controller for our application is definitely advisable as it avoids all the delays and the discontinuities in wireless connections. A miniature computer module (CM), based on Geode 1200 processor running at $266 \mathrm{Mhz}$ with 128 Mo of RAM and as much of flash memory was developed. The computer module is $\mathrm{x} 86$ compatible and offers all standard $\mathrm{PC}$ interfaces in addition to an $\mathrm{I}^{2} \mathrm{C}$ bus port. The whole computer is $44 \mathrm{~g}$ in mass, $56 \mathrm{~mm}$ by $71 \mathrm{~mm}$ in size (see Fig. 5) and runs a Debian based minimalist Linux distribution.

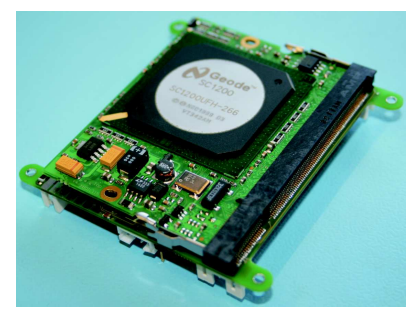

Fig. 5. The $\mathrm{x}$-board based, $40 \mathrm{~g}$ and $56 \times 71 \mathrm{~mm}$ computer module.

\section{The Communication Modules}

The controller described in the paragraph above includes an MCU for Bluetooth chip interfacing with the computer module. The same MCU is used to decode the $\mathrm{PPM}^{7}$ signal picked-up from a $1.6 \mathrm{~g}, 5$ channels commercially available $\mathrm{RC}$ receiver. This makes it possible to change the number of channels as convenient and control the robot using a standard remote control. Finally, a wireless LAN USB adapter was added. On the ground side, a standard GCS ${ }^{8}$

\footnotetext{
7 Pulse Position Modulation

${ }^{8}$ Ground Control Software
} 
for all our flying robots is developed. Presently, it permits UAV environment visualization, waypoints and flight plans management as well as data logging and controller parameters tuning.

\section{The Design Results}

The robot as a whole represents the result of the design methodology and fits the requirements. One can see mass and power distributions from Fig. 6 . The total mass is about $520 \mathrm{~g}$ where the battery takes almost the onehalf and the actuators only the one-third thanks to BLDC technology. All the actuators take obviously the lion's part, $60 \mathrm{~W}$ of $66 \mathrm{~W}$ the total power consumption. However, the latter depends on flight conditions and represents a weighted average value between the equilibrium $(40 \mathrm{~W})$ and the worst possible inclination state $(120 \mathrm{~W})$ without loosing altitude. Fig. 7 shows the real robot.
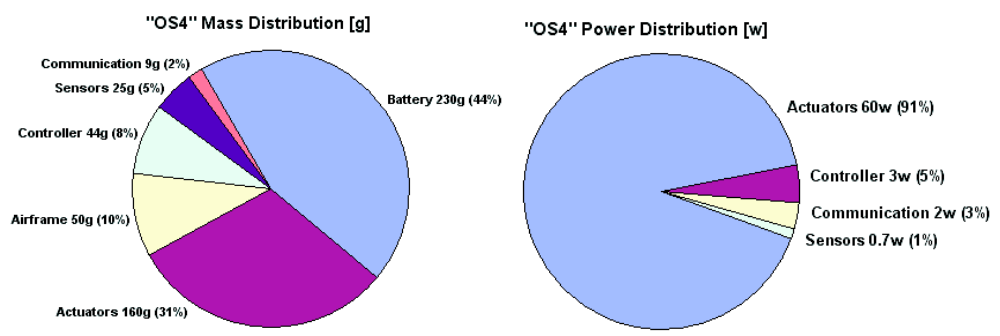

Fig. 6. Mass and power distributions in "OS4" robot.

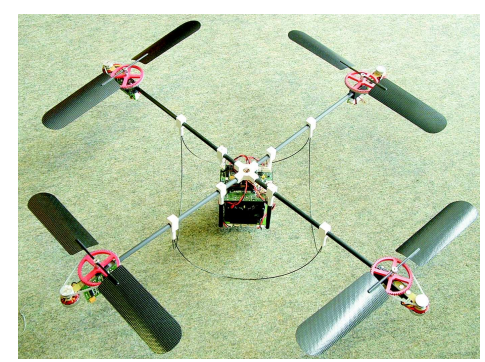

Fig. 7. The "OS4" quadrotor. 


\section{Modelling}

Modelling a helicopter is a quite complex task and one has to make some simplifying. In this case, the airframe is rigid, all the propellers are in the same horizontal plan and the quadrotor structure is symmetric. Obviously, only the dominant effects are modelled. The dynamics of a rigid body under external forces applied to the center of mass and expressed in the body fixed frame as shown in [9] are in Newton-Euler formalism:

$$
\left[\begin{array}{cc}
m I_{3 \times 3} & 0 \\
0 & I
\end{array}\right]\left[\begin{array}{c}
\dot{V} \\
\dot{\omega}
\end{array}\right]+\left[\begin{array}{c}
\omega \times m V \\
\omega \times I \omega
\end{array}\right]=\left[\begin{array}{c}
F \\
\tau
\end{array}\right]
$$

Where $I \in \Re^{(3 \times 3)}$ the inertia matrix, $V$ the body linear speed vector and $\omega$ the body angular speed. Let's consider $U_{1}, U_{2}, U_{3}, U_{4}$ as the system inputs and $\Omega$ as a disturbance:

$$
\left\{\begin{array}{l}
U_{1}=b\left(\Omega_{1}^{2}+\Omega_{2}^{2}+\Omega_{3}^{2}+\Omega_{4}^{2}\right) \\
U_{2}=b\left(-\Omega_{1}^{2}-\Omega_{2}^{2}+\Omega_{3}^{2}+\Omega_{4}^{2}\right) \\
U_{3}=b\left(-\Omega_{1}^{2}+\Omega_{2}^{2}+\Omega_{3}^{2}-\Omega_{4}^{2}\right) \\
U_{4}=d\left(\Omega_{1}^{2}-\Omega_{2}^{2}+\Omega_{3}^{2}-\Omega_{4}^{2}\right) \\
\Omega=-\Omega_{1}+\Omega_{2}-\Omega_{3}+\Omega_{4}
\end{array}\right.
$$
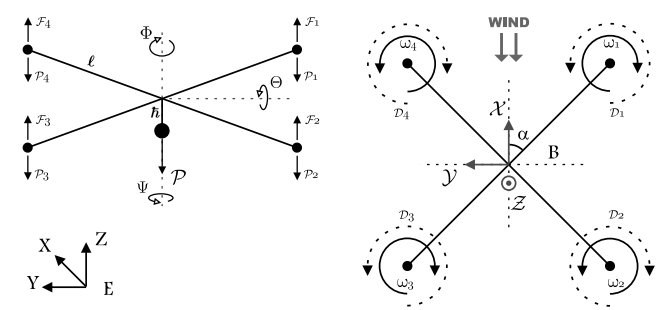

Fig. 8. The "OS4" coordinate system.

\subsection{Moments Acting on a Quadrotor}

\section{Actuators Action}

Several combinations of propellers actions are possible for rolling or pitching a quadrotor. Following the coordinate system on Fig. 8, one can write:

$$
\tau_{a}=\left(\begin{array}{c}
l \cos \alpha U_{2} \\
l \cos \alpha U_{3} \\
U_{4}
\end{array}\right)
$$

The first two elements of (3) include the $\Delta T=\Sigma T_{i}$ and the third one the $\Delta D=\Sigma D_{i}$ aerodynamic effect listed in Table 2 . 


\section{Rotors Gyroscopic Effect}

One of the most important sources of instability in a quadrotor. One can attenuate it by reducing the propellers rotational speed or inertia. The dumping also increases by lowering the CoG. Otherwise, one can constrain the control to keep it compensated between each pair of propellers.

$$
\tau_{p}=\left(\begin{array}{c}
J_{r} \dot{\theta} \Omega \\
J_{r} \dot{\phi} \Omega \\
0
\end{array}\right)
$$

\section{Rotors Inertial Counter Torque}

These terms result from the reaction torque produced by a change in rotational speed [10].

$$
\tau_{i}=\left(\begin{array}{c}
0 \\
0 \\
J_{r} \dot{\Omega}
\end{array}\right)
$$

\section{Horizontal Motion Friction}

The friction force on the propellers resulting from horizontal linear motion induces moments on the helicopter body. The $F_{x, y}$ forces depend on $V$ and $\Omega_{i}$ and must be estimated.

$$
\tau_{f}=\left(\begin{array}{c}
F_{x} h \\
F_{y} h \\
0
\end{array}\right)
$$

The moments due to propeller lift dissymmetry are neglected thanks to "OS4" construction (see, Fig. 4). From (1) - (6) one can rewrite the quadrotor rotational dynamics:

$$
\left[\begin{array}{c}
I_{x x} \ddot{\phi} \\
I_{y y} \ddot{\theta} \\
I_{z z} \ddot{\psi}
\end{array}\right]=\omega \times I \omega+\tau_{p}+\tau_{a}+\tau_{i}-\tau_{f}
$$

\subsection{Forces Acting on a Quadrotor}

\section{Actuators Action}

The quadrotor is an underactuated system hence it's horizontal motion is mainly due to the orientation of the total thrust vector (using the rotation matrix).

$$
F_{a}=\left(\begin{array}{c}
\cos \phi \sin \theta \cos \psi U_{1}+\sin \phi \sin \psi U_{1} \\
\cos \phi \sin \theta \sin \psi U_{1}-\sin \phi \cos \psi U_{1} \\
-m g+\cos \phi \cos \theta U_{1}
\end{array}\right)
$$




\section{Horizontal Motion Friction}

The friction force on vehicle's body during horizontal motion is:

$$
F_{f}=-C_{x, y, z} V^{2}
$$

From (1), (2), (8) and (9) one can rewrite the quadrotor translational dynamics:

$$
\left[\begin{array}{c}
m \ddot{x} \\
m \ddot{y} \\
m \ddot{z}
\end{array}\right]=\omega \times m V+F_{a}-F_{f}
$$

\section{3 "OS4" Model Parameters}

Table 3 lists most of "OS4" model parameters. The inertia matrix is supposed diagonal thanks to the symmetric construction. The CAD software gives the exact inertia values. The remaining aerodynamic parameters will be identified in near future.

Table 3. "OS4" Main Model Parameters.

\begin{tabular}{c|c|c|c}
\hline parameter & value & unit & \\
\hline thrust coef. & $b$ & $3.13 \mathrm{e}-5$ & $\mathrm{~N} \mathrm{~s}^{2}$ \\
drag coef. & $d$ & $7.5 \mathrm{e}-7$ & $\mathrm{Nm} \mathrm{s}^{2}$ \\
inertial moment on $x$ & $I_{x x}$ & $6.228 \mathrm{e}-3$ & $\mathrm{~kg} \mathrm{~m}^{2}$ \\
inertial moment on $y$ & $I_{y y}$ & $6.225 \mathrm{e}-3$ & $\mathrm{~kg} \mathrm{~m}^{2}$ \\
inertial moment on $z$ & $I_{z z}$ & $1.121 \mathrm{e}-2$ & $\mathrm{~kg} \mathrm{~m}^{2}$ \\
arm length & $l$ & 0.232 & $\mathrm{~m}$ \\
CoG to rot. plane & $h$ & $2.56 \mathrm{e}-2$ & $\mathrm{~m}$ \\
robot mass & $m$ & 0.52 & $\mathrm{~kg}$ \\
propeller inertia & $J_{r}$ & $6 \mathrm{e}-5$ & $\mathrm{~kg} \mathrm{~m}^{2}$ \\
\hline
\end{tabular}

\section{Simulation}

Several simulations were performed under Matlab using the model parameters listed in Table 3 with a simple PD controller (Roll and Pitch: $\mathrm{Kp}=1, \mathrm{Td}=0.6$. Yaw: $\mathrm{Kp}=0.4, \mathrm{Td}=0.3)$. The task was to stabilize the helicopter attitude to $(\phi=\theta=\psi=0)$, from $(\phi=\theta=\psi=\pi / 4)$ initial conditions. The simulated performance was satisfactory as showed in Fig. 9. 

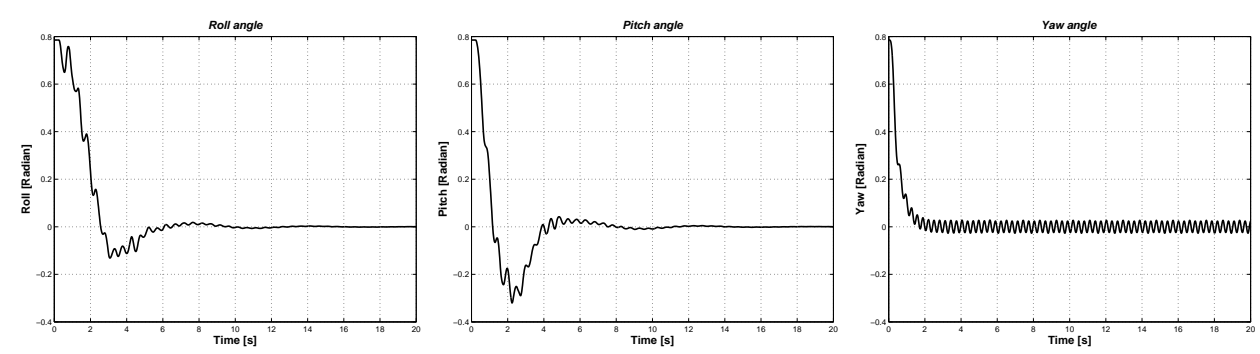

Fig. 9. Simulation: The PD controller has to stabilize the attitude.

\section{Experiment}

We tested successfully a real flight experiment using only the IMU sensor for attitude control (Roll and Pitch: $\mathrm{Kp}=0.8, \mathrm{Td}=0.3$. Yaw: $\mathrm{Kp}=0.08, \mathrm{Td}=0.03$ ). The robot exhibits the predicted thrust. However, the motor module bandwidth seem to be slow, this is partly responsible for the oscillations in Fig. 10. A new version of the motor module is under development. The experimental results are considered satisfactory as they practically validate part of the system in real operation.
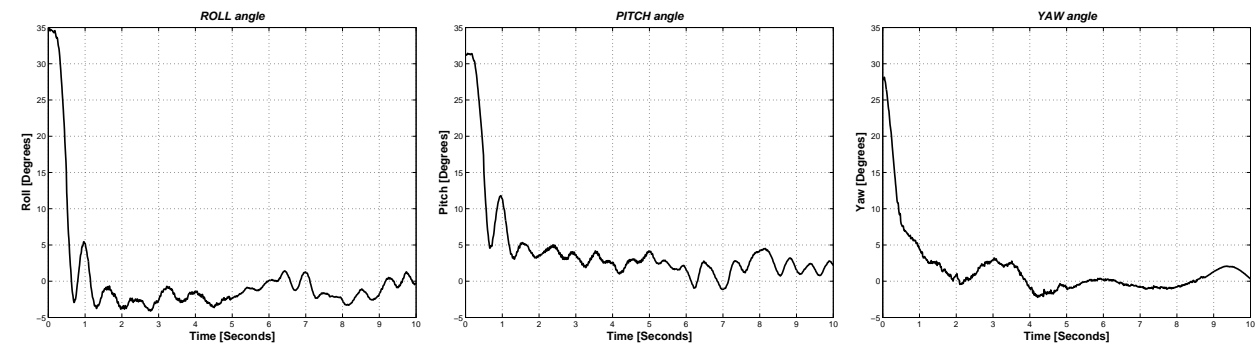

Fig. 10. Experiment: The first test flight with a PD controller. The stabilization is satisfactory.

\section{Conclusion}

This paper presented a practical method for miniature rotorcraft design. It was the only tool used to get the satisfied design requirements and achieve the excellent $100 \%$ thrust margin for 30 min autonomy. Our quadrotor embeds all the necessary avionics and energy devices for a fully autonomous flight. We derived the nonlinear dynamic model with accurate parameters, performed a simulation and successfully realized a test flight. The future goal is the 
implementation of the control strategies developed for the first prototype at the beginning of the "OS4" project. Most parts of this development are for indoor as well as outdoor environments with minor adaptations. The numerous innovations and design results presented in this paper reinforce our conviction in the emergence of miniature intelligent flying platforms.

\section{Acknowledgement}

The authors would like to thank André Noth for fruitful discussions about flying robots, André Guignard for the mechanical parts realization, Peter Bruehlmeier for PCB design and all the students who worked or are working on the project.

\section{References}

1. Floreano D, Zufferey J.C. and Nicoud J.D. (2005) Artificial Life Winter-Spring 2005:121-138

2. Pounds P, Mahony R, Gresham J, Corke P, Roberts J (2004) Towards Dynamically-Favourable Quad-Rotor Aerial Robots. Australasian Conference on Robotics and Automation, Canberra, Australia

3. Ruffier F, Franceschini N (2004) Visually Guided Micro-Aerial Vehicle: Automatic Take Off, Terrain Following, Landing and Wind Reaction. IEEE International Conference on Robotics and Automation, New Orleans, USA

4. Kroo I, Prinz F, Shantz M, Kunz P, Fay G, Cheng S, Fabian T, Partridge C (2000) The Mesicopter: A Miniature Rotorcraft Concept Phase II Interim Report. Stanford University, USA

5. Bouabdallah S, Siegwart R (2005) Backstepping and Sliding-mode Techniques Applied to an Indoor Micro Quadrotor. IEEE International Conference on Robotics and Automation, Barcelona, Spain

6. Bouabdallah S, Murrieri P, Siegwart R (2004) Design and Control of an Indoor Micro Quadrotor. IEEE International Conference on Robotics and Automation, New Orleans, USA

7. Prouty R.W (2002) Helicopter Performance, Stability, and Control. Krieger Publishing Company

8. Bouabdallah S, Murrieri P, and Siegwart R (2003) Autonomous Robots Joural Mars 2005

9. Sastry S (1994) A mathematical introduction to robotic manipulation. Boca Raton, FL

10. Müllhaupt P (1999) Analysis and control of underactuated mechanical nonminimum-phase systems. PhD Thesis, EPLF, Switzerland

11. Olfati-Saber R (2001) Nonlinear control of underactuated mechanical systems with application to robotics and aerospace vehicles. PhD Thesis, MIT, USA 